\title{
Tangence
}

\section{Mémoire, deuil et intertexte dans La boucle de Jacques Roubaud}

\section{Anne Roche}

Numéro 45, octobre 1994

Authenticité et littérature personnelle

URI : https://id.erudit.org/iderudit/025826ar

DOI : https://doi.org/10.7202/025826ar

Aller au sommaire du numéro

Éditeur(s)

Tangence

ISSN

0226-9554 (imprimé)

1710-0305 (numérique)

Découvrir la revue

Citer cet article

Roche, A. (1994). Mémoire, deuil et intertexte dans La boucle de Jacques

Roubaud. Tangence, (45), 74-82. https://doi.org/10.7202/025826ar d'utilisation que vous pouvez consulter en ligne.

https://apropos.erudit.org/fr/usagers/politique-dutilisation/ 


\section{Mémoire, deuil et intertexte dans La boucle de Jacques Roubaud}

\section{Anne Roche}

La boucle, de Jacques Roubaud ${ }^{\mathbf{1}}$, ne se présente pas comme un journal: publié dans la collection Fiction et Cie, qui s'ouvre à des textes de prose ou parfois de poésie mais toujours présentant un caractère de recherche, voire de laboratoire, le texte, qui ne comporte pas d'indication générique de couverture (telle que roman ou récit) se subdivise intérieurement en un Récit de six chapitres, eux-mêmes formés de cinquante textes plus ou moins courts, suivi d'une seconde partie d'Insertions qui se greffent sur chacun des six chapitres, les Insertions numérotées de 51 à 131, et enfin d'une troisième partie de Bifurcations, regroupées en six sections (de A à F) et en textes numérotés de 132 à 196. Une telle organisation, ici sommairement décrite, ne peut que s'opposer à ce que semble impliquer la notion même de journal: texte formé par l'accumulation des entrées quotidiennes, n'ayant de structure que celle, aléatoire, de la succession des jours, pouvant posséder des logiques thématiques, psychiques, etc. mais en aucun cas une architecture concertée, sous peine de se nier soi-même en tant qu'écriture diaristique.

Étudier néanmoins ce texte dans le cadre d'un numéro consacré à l'écriture personnelle et à la question de l'authenticité ne relève pas du paradoxe ou du décalage. Si en effet on examine le dispositif qui se met en place entre trois des dernières ouvres de Roubaud (Quelque chose noir ${ }^{2}$, qualifié de "poèmes", Le grand incendie de Londres ${ }^{3}$, qualifié de "récit avec incises et bifurcations", et La boucle déjà cité), on constate qu'en dépit de la contradiction générique clairement affichée par l'indication de couverture, dans le cas des deux premiers, ou par la table des matières pour le dernier, ils entretiennent des rapports forts avec l'écriture du moi, voire du journal, qu'ils posent des questions au

\footnotetext{
1 Jacques Roubaud, La boucle, Paris, Seuil, 1993.

2 Paris, Gallimard, 1986.

3 Paris, Seuil, 1989.
} 
cœur de cette écriture, et que, tout en s'en démarquant de façon formelle, ils sont de nature à nous apporter des clartés sur un fonctionnement qu'ils semblent exclure.

\section{Distance temporelle et authenticité : le paradoxe de la fleur de gel}

On le sait: le propre de l'écriture de journal est son immédiateté. Le diariste écrit tous les soirs ce qui lui est arrivé dans la journée, même s'il lui arrive de "rattraper" avec quelque artifice le retard qu'il a pu prendre dans sa relation, replâtrage dont le journal fictif tenu dans L'emploi du temps de Butor ${ }^{4}$ donne la version maniaque et angoissée. À quoi s'oppose l'écriture de l'autobiographe ou du romancier, qui prend le temps de la réflexion, ce qui veut dire de la construction: contraste fort intéressant à observer quand nous disposons des deux versants de l'activité d'un même écrivain, comme c'est le cas par exemple avec Michel Leiris ${ }^{5}$. Or, ce décalage temporel pose entre autres la question de la fiabilité du souvenir, de son authenticité. Les pages inaugurales de $L a$ boucle, à partir d'un souvenir d'enfance qui pourrait être anodin (l'enfant observant les fleurs que le gel dessine sur la vitre nocturne), dëplient toutes les impossibilités logiques du souvenir, interdisent au lecteur le plaisir simple de la jouissance et de l'identification pour lui ouvrir les plaisirs plus austères d'une théorie de la mémoire, ou plutôt de ses apories. Incertitudes sur la date, anesthésie de la sensation que l'adulte relate par savoir analogique et non parce qu'il y aurait sensation réelle, bref, "la moindre ligne du récit de ce souvenir contient une énorme quantité de conclusions implicites $" 6$.

Pour rendre compte de ce caractère insaisissable et éphémère du souvenir, le scripteur se propose "d'écrire des mots, des signes, immédiatement effaçables " 7 . Tel est du moins l'art poétique mis en avant, mais ce mimétisme est impossible - est-il même souhaité par l'écrivain? - : l'écriture, elle, est durable, veut

4 Paris, Minuit, 1956.

5 Les autobiographies de L'âge d'Homme (Paris, Gallimard, 1939) et de La règle du jeu (Paris, Gallimard, 1948 à 1976) se sont nourries de la tenue du Journal (Paris, Gallimard, 1992), lequel pourtant ne nous a été connu que par une publication posthume).

6 La boucle, op.cit., p. 12.

7 Ibid., p. 13. 
durer. Mais sa durée est d'un autre ordre que celle du souvenir, ce que le journal, précisément, tend à confondre ou plutôt à masquer, alors que Roubaud, lui, démasque ce fonctionnement illusoire de l'écriture:

L'image du passé (et, en fait, toute image est du passé), dite souvenir, n'a pas de durée. Elle vient au monde [...] sans légende, sans mode d'emploi, sans explications. [...] Dès qu'on s'arrête un peu sur elle, au lieu de l'accueillir sans hésitation, comme disant le vrai du passé, comme nous apportant un savoir sur le passé qui commanderait une croyance raisonnable en lui, et qu'on s'interroge sur cette non-durée du souvenir, on ne peut qu'être saisi de doute. ${ }^{8}$

\section{Le présent de l'écriture}

À partir de ce doute, il n'est peut-être légitime que d'écrire du présent, et au présent. Le présent de l'écriture nervure La boucle et Le grand incendie de Londres. L'incipit de ce dernier texte ressemble à une entrée canonique de journal:

\section{Ce matin du 11 juin 1985}

Ce matin du 11 juin 1985 (il est cinq heures), pendant que j'écris ceci sur le peu de place laissé libre par les papiers à la surface de mon bureau, j'entends passer, dans la rue des Francs-Bourgeois, deux étages plus bas...?

et tout le premier chapitre, "La lampe", décrit l'activité d'écriture, de recopiage, de mise au net..., description qui pourrait paraitre fastidieuse au lecteur si, très vite, elle n'était en quelque sorte sommée par une motivation encore énigmatique, mais qui aiguillonne la lecture: "dans cet intervalle quotidien de ma vie maintenant vide, j'écrirai". Que signifie "maintenant vide"? Si le lecteur ignore tout du hors-texte, c'est-à-dire de la biographie de Jacques Roubaud, ou des textes antérieurs, peu importe, car l'énigme sera levée peu après: "cet autre sommeil, et chaleur à la fois intense et troublée, celle de ma jeune femme endormie. [...] Et mon silence est inutile, puisqu'elle est morte. ${ }^{10}$ Le présent d'écriture se dévoile donc comme partiellement illusoire: des ges-

\footnotetext{
8 Ibid., p. 15-16.

9 Le grand incendie de Londres, op. cit., p. 13.

10 Ibid., p. 50
} 
tes ont été répétés, comme s'ils avaient le sens du passé, (écrire au coeur de la nuit, ne pas faire de bruit pour ne pas réveiller l'endormie) mais ce faux présent, malgré les rituels qu'il met en place, ne peut éliminer ce sur quoi il est fondé, la connaissance du passé (elle est morte).

La question peut paraître trop spécifique - même si l'on défend l'idée que toute écriture prend origine dans une perte. Mais, sans tomber dans les caractérologies toujours suspectes qui assignent aux auteurs de journaux tel ou tel trait particulier, reste que l'activité d'écriture décrite par Roubaud dans ce premier chapitre du Grand incendie, par son caractère obsessionnel, engendre un sentiment d'angoisse qui se communique aisément au lecteur, même si celui-ci n'est pas encore averti de la perte réelle qui l'a déclenchée, et crée donc une forte impression d'authenticité. Impression renforcée par les protocoles qui entourent le geste d'écrire, et qui se veulent ritualisés, inchangeables, comme c'est souvent le cas pour les diaristes:

Je voudrais en somme conserver quasi immuables les conditions d'une expérience le plus possible quotidienne de prose; que le lieu en soit presque invariable, le temps fixe; que les signes que j'écris, et qui s'ajoutent, se poussent l'un l'autre dans mon cahier, s'arrêtent sur cette image de quasi-perrnanence... ${ }^{11}$

À ces conditions du geste s'ajoute, implicite mais non moins net, l'engagement tacite de ne pas détruire et de ne pas modifier l'ordre des lignes et des signes, ce qui est encore une des caractéristiques de l'écriture diaire.

\section{Écrire pour détruire}

Mais cet engagement de ne rien détruire du texte a un envers apparemment inattendu, qui se situe du côté du désir de mort du scripteur :

Dans le segment de nuit finissante, qui mord d'un côté sur la boue de mon sommeil, de l'autre sur le déroulement ordinairement désertique de mes journées, chaque fragment de mémoire que j'extirperai du temps, aussitôt posé noir ici, s'évaporera, comme la lumière posée jaune par la lampe devant la plus décidée lumière qui est celle du jour.

11 Ibid., p. 14. 
78

Ce qui restera sera cette narration; entrelacée à la nuit, à son mauvais silence; où j'espère, par l'accumulation et la persévérance, parvenir, ne serait-ce qu'involontairement, à ma fin. ${ }^{12}$

Dès lors, la répétition des gestes nous apparaît sous un jour plus sinistre: le texte crée l'illusion d'optique d'un présent éternel, peut-être celui de la réitération du moment traumatique:

Cette image se présente pour la millième fois à neuf avec la même violence elle ne peut pas ne pas se répéter indéfiniment une nouvelle génération de mes cellules si temps il y a trouvera cette duplication onéreuse ces tirages photographiques internes je n'ai pas le choix maintenant ${ }^{13}$,

réitération qui est à la fois très différente de la répétition diaire (répétition du quotidien, du banal, de l'habituel), puisqu'il s'agit là de l'irruption de l'exceptionnel, de ce qui par définition n'a lieu qu'une fois (la mort de l'aimée), mais qui en même temps s'y apparente, parce que le travail du deuil ne se peut que par ce retour du même.

Telle est du moins la situation dans Quelque chose noir et Le grand incendie de Londres. Dans La boucle, une différence majeure apparaît dans la tonalité : le deuil semble en bonne voie (en tout cas le deuil d'Alix), ce qui, curieusement ou non, permet à d'autres morts d'émerger: la mort du jeune oncle Frantz en 1938 (mort dont on ne nous dit rien, sinon qu'elle ébranla durablement la famille ${ }^{14}$ ), et la mort par suicide du jeune frère ${ }^{15}$. Deuils asymétriques: le deuil du frère est ce qui fait advenir à l'écriture, le deuil d'Alix est ce qui l'interdit:

Devant ta mort je suis resté entièrement silencieux.

Je n'ai pas pu parler pendant presque trente mois.

Je ne pouvais plus parler selon ma manière de dire qui est la poésie.

J'avais commencé à parler, en poésie, vingt-deux ans avant.

C'était après une autre mort.

Avant cette autre mort je ne savais comment dire, j'étais comme silencieux. Ainsi, pris entre deux "bords" de mort ${ }^{16}$

12 Ibid., p. 15

13 Quelque cbose noir, op. cit., p. 11.

14 La boucle, op. cit., p. 75.

15 Évoquée dans quelques poèmes d'E (Paris, Gallimard, 1967).

16 Quelque chose noir, op. cit., p. 131-132. 
Mais les morts de La boucle sont à peine objet d'allusion: le sujet du livre, c'est la mémoire, et une recherche du paradis perdu (dit comme tel) que fut Carcassonne, mais aussi une recherche des origines, de "ce qui a fait de moi ce que je suis", à la fois les parents, les amis, leurs faits et gestes, et les livres.

\section{De la mémoire enfantine à l'histoire historique}

Il y a dans La boucle une euphorie de la mémoire corporelle, une jouissance à reconstituer physiquement les trajets enfantins, les sensations: "à genoux devant le banc vert, les genoux nus" 17 Roubaud se livre à l'élaboration d'une véritable charte du souvenir, des trajets mémoriels, de leurs difficultés mais aussi de leur charme. Le souvenir concret s'ancre à l'invention de mots (Oranjeaunue $)^{18}$ : l'enfance est l'époque des apprentissages, où tout semble possible, où le réservoir des mots est illimité, dès lors, pourquoi ne pas y ajouter? ${ }^{19}$ Les noms propres, comme chez Proust, ont aussi un pouvoir évocateur:

J'écris: impasse des Mûriers ${ }^{20}$

Mais avant tout, le souvenir est affaire de transmission familiale. Roubaud se propose d'étudier les connexions familiales et amicales, d'en faire l'histoire; mais la transmission familiale n'est jamais systématique, et s'amarre le plus souvent à des anecdotes insignifiantes: ainsi de Simone Weil, nous apprenons surtout qu'elle a offert un jeu de quilles au petit Jacques... Aussi le chroniqueur doit-il compléter son information par le recours à d'autres sources, surtout lorsque ses "informateurs" sont morts pour la plupart: livre d'histoire contemporaine ${ }^{21}$ où il découvre des renseignements inédits pour lui sur son père et son oncle Frantz, carnets tenus par le grand'père, lettre du petit Jacques à ses parents, etc. Mais - là intervient son souci de véridicité — il ne lisse pas son discours: par la typographie et par les sertissures, il nous donne à voir ses différentes sources, livresques,

17 La boucle, op. cit., p. 95.

18 Ibid., p. 130.

19 Il est à noter que Roubaud comme poète ne fait pas de néologismes, par exemple de mots-valises comme Leiris : sa novation se situe plutôt au niveau de la syntaxe.

20 Ibid., p. 47.

21 Ibid, p. 76-77. 
rapportées de vive voix, ou expérience personnelle, et il permet au lecteur de les différencier pour faire ses propres montages.

Ce recours au passé ne contredit donc pas l'affirmation: "Je n'ai pas de curiosité généalogique "22. Peut-être d'ailleurs est-ce un des points qui différencie Roubaud de Perec: Perec orphelin avait une grande "curiosité généalogique", et projetait d'écrire L'arbre ou Le livre d'Esther, livre qui aurait constitué son "arbre" généalogique depuis la Pologne native de ses parents: il questionnait pour cela sa tante Esther, seule survivante de sa famille. Roubaud, vivant dans une famille heureuse, unie, soudée de plus par une disparition douloureuse certes mais non destructrice (celle de Frantz), n'éprouve pas le même besoin. Ce qui vérifierait notre hypothèse, c'est qu'il parle davantage du côté paternel de sa généalogie, justement parce que "de l'autre côté, (paternel) il n'y avait que des absences, énumérées, comme autant de pierres tombales, par des noms " 23 .

Mais cette recherche du passé ou de la généalogie ne se limite pas à la famille, et c'est là peut-être que se lit le paradoxe le plus flagrant de La boucle: dans ce rapport avéré que le livre entretient aux textes antérieurs, rapport qui s'avoue usuellement dans les thèses, les essais, les ouvrages scientifiques, mais qui est généralement masqué, voire inconscient, dans les textes de fiction, comme si la citation ou la référence venait briser l'illusion de l'authentique. Ici encore, Roubaud prend le contrepied de la pratique habituelle: dans $L a$ boucle, les intertextes sont multiples, dans leur choix, et dans leur insertion, et dans leur fonction. Autocitations, qui se subdivisent en citations de l'auteur quand il n'est pas encore un auteur (sa lettre de petit garçon à ses parents) ${ }^{24}$, citations de l'auteur devenu un auteur, massives ${ }^{25}$, et dans ce cas l'auto-citation cherche à reconstruire une continuité perdue, ou bien discrètes: ainsi le mot "hirsute", présenté comme vocable emprunté à une règle rhétorique, appuyée sur l'autorité de Dante,

22 Ibid., p. 79.

23 Ibid.

24 Ibid., p. 178.

25 "Saint-Jean mil neuf cent trente neuf" (p. 160-161) poème d' $E$ sur lequel il se livre à une "prosification", ou "Place Davila" (p. 153): le poème arrive pour "clôturer" une longue exégèse en prose qui le précède, mais l'auteur ne sait si c'est pour l'éclairer ou "l'obscurcir définitivement ". Ces poèmes, tous deux extraits d' $E$, sont parmi les plus anciens du recueil, donc particulièrement liës à l'enfance, à la thématique même de La boucle. 
Pétrarque, Arnaut Daniel ${ }^{26}$. En fait, si on a lu Quelque chose noir, on se rappelle un poème intitulé "Pexa et hirsuta" (peignée et hirsute) qui pivote de Dante à une évocation érotique de la nudité d'Alix et de son nom fragmenté ${ }^{27}$. La figure d'Alix a disparu de ce texte-ci (elle est présente ailleurs) mais l'éros mélancolique y est, sous la forme d'un amour impossible du gamin Roubaud, amour dont l'objet se tuera peu après dans un accident ${ }^{28}$. De tels amarrages assurent la cohésion du texte, qui peut être de l'ordre de l'obsessionnel: on verra plus loin que l'intertextualité a une autre fonction qui est au contraire de mettre en doute, non le texte, mais une attitude naiive du lecteur en face de celui-ci.

Nombreuses sont aussi les citations d'autrui: citations d'auteurs classiques, reconnus, mais à des degrés différents, banales, parfois "transfigurees " par le bricolage familial:

Des meubles luisants

Polis par les dents

Décoreraient notre chambre... ${ }^{29}$

citations plus rares, parfois cryptées dans un texte précédent et ici dévoilées ${ }^{30}$. Mais aussi citations d'"écrivants " d'occasion: extraits du journal du grand'père, documents divers, le sauf-conduit portant les diverses identités du père et... signé par lui-même, le témoignage de la Juive sauvée par la grand'mère, la reconnaissance officielle et posthume de la qualité de "Juste" pour celle-ci, graffitti anonymes relevés dans le métro. Dans cette famille cultivée, même les canards font des citations: si la cane pond son ouf en évidence sur le fauteuil de la salle à manger, c'est bien sûr qu'elle a lu et "sérieusement médité " La lettre volée ${ }^{31}$...

De telles insertions, et si nombreuses, marquées en outre par des différences typographiques, ne nuisent-elles pas à la crédibilité du texte, ou du moins n'empêchent-elles pas le lecteur de lire naïvement? Certes, elles ont pour effet d'interdire le "confort autobiographique ", qui consiste pour Roubaud à coaguler frauduleusement le passé, le présent et le futur ${ }^{32}$ : elles nous rappellent

26 Ibid., p. 231.

27 Quelque chose noir, op. cit., p. 64.

28 La boucle, op. cit., p. 232.

29 Ibid., p. 106.

30 Ibid., p. 330-331.

31 Ibid., p. 121.

32 Ibid., p. 62. 
82

que, de même qu'un enfant naît de ses parents, de ses aïeux, le texte naît des livres lus et n'est pas un objet naturel que nous puissions consommer comme tel. Mais précisément, toute la stratégie, si retorse, de Roubaud, tend au même but: qu'il s'agisse de démonter les apories de la mémoire, ses sophismes, d'écrire un deuil dont la sincérité ne fait aucun doute, ou de tracer les origines du texte et du moi, tous les éléments du texte démontent l'illusion référentielle, pour laisser le lecteur confronté à un questionnement plus inquiétant. "L'autobiographie, ironise Roubaud, genre qui est un des derniers refuges du déterminisme mécaniste " ${ }^{33}$ : même s'il n'est pas entièrement exempt des ruses narratives qu'il dénonce chez les autres autobiographes, notamment Sartre et Leiris, il est parvenu, tout en écrivant un texte profondément personnel et impliqué, à éviter ce piège de l'autobiographie où tout s'explique, où ne subsiste plus rien d'obscur, plus rien de ce quelque chose noir auquel chacun est tôt ou tard confronté.

33 Ibid., p. 163. 